\title{
Bearing Capacity Estimates of the Parallel Wire Cables of Large Span Cable-stayed Bridges
}

\author{
Bing BAI ${ }^{1} \mathrm{a}^{*}$, Xiao-Long $\mathrm{LI}^{1}$, Yue-Ling $\mathrm{LI}^{1}$ \\ ${ }^{1}$ Bridge Technology Research Center, Research Institute of Highway Ministry of Transport, No.8 \\ Xitucheng Road, Beijing, China \\ a714840826@qq.com \\ ${ }^{*}$ Corresponding author
}

\begin{abstract}
Keywords: Parallel Wire Cable, Bearing Capacity, Wire Tensile Strength, Length Effect, Daniels Model, Wire Correlation Length, Daniels Effect.
\end{abstract}

\begin{abstract}
As the stay cables are the key members of large span cable-stayed bridges, Accurate calculation of its bearing capacity is the basic foundation of structure design and soundness assessment. Using the statistical theory, $81 \varphi 7-1770 \mathrm{MPa}$ new wire strength test results are employed to investigate the law of wire tensile strength distribution. Based on this, the cable parallel system and Monte Carlo sampling method are adopted to develop the Daniels model based bearing capacity calculation theory of parallel wire cable. Using this model, the general law between cable bearing capacity and wire number in it is investigated further. The research indicates that the tensile strength of single short wire follows the normal distribution. And the tensile strength decreases with the increase of the wire length. The whole bearing capacity per single wire also decreases with the increase of wire number in the cables.
\end{abstract}

\section{Introduction}

Efficient use of building material and excellent mechanical behavior have made construction of cable-stayed bridge a significant trend of long-span bridge solution across the navigable rivers. For this type of bridges, dense cable system is a remarkable symbol of structure modernization. The strength of them could affect the whole structure safety greatly, which is proved by mass bridge crash events repeatedly [1]. In view of this fact, there still exists urgent demand for the establishment of rational cable strength assessment methodology. The research on that is of great significance for both bridge design and constructions.

According to the current technology, there are only two kinds of stay cables used in actual structures: one is steel stand cable, another is parallel wire cable. Compared with the former, the parallel wire cable is more advantageous on manufacturing quality, uniform tensioning and construction. As a result this kind of cable is more popular in application of China bridge construction programs. Studies on this issue may be more universally available to the design and health assessment of structures.

For parallel wire cables, it has been widely accepted that the static wire strength has to be modeled by random variables. Hence the resistance model (also known as bearing capacity model) of it in actual is a probabilistic mechanical model. From this point of view, Faber [2] and Lan [3] both proposed a cable (wire bundles) resistance model on account of the Daniels probabilistic strength theory. In their models, the wire resistances are all assumed to follow Weibull distribution as a basis. However, both Faber and Lan didn't discuss the validation of this assumption in some detail, which makes the hypothesis questionable. Similarly with above, Sante Camo [4] adopted normal distribution assumption of wire strength, and analyzed both "brittle wire model" and "ductile wire model" in comparison to experimental data separately. The result indicates that the brittle wire model seems to be more consistent with the test data. Different from these ideas, Cremona [5] and Zhu [6] developed a Monte Carlo-based method for the assessment of cable bearing capacity with the use of wire strength test data. This approach is rather promising and may lead to another methodology for the evaluation of cable strength. According to these solid researches, both wire failure mode and cable bearing capacity have been investigated comprehensively, which achieved the cable strength evaluation theory greatly. 
In spite of the excellent studies on cable resistance above, less work has so far been presented on account of the experiment and specimen amount restriction. The previous studies barely discussed the distribution type and parameters of wire strength. The issue, however, is the key foundation for the estimate of cable strength. Developing a model based on accurate wire strength distribution will be more rational and practical. On account of all these, the present paper attempts to discuss the wire strength distribution as well as whole cable bearing capacity assessment. By the use of 81 wire tensile tests and statistical theory, the probability distribution of wire strength will be discussed firstly. Then with the Daniels probabilistic strength theory and Monte Carlo method, the bearing capacity of parallel wire cables will be investigated further.

\section{Probabilistic Model of Single Wire Strength}

Different from pylon and stiffening girder members, the actual stay cable is very long and slender. Hence individual wires in it may be considered as being a weakest link structural system (a series system) [2,7]. The strength of it is actually determined by the weakest segment, see Fig. 1. For experimental restrictions, testing this long wire is obviously unrealistic. The current operation specification generally demands the specimen as short as $0.25 \mathrm{~m}$ in length [8]. As can been seen easily from above, this testing method can hardly reflect the influence of series system on wire strength. Hence the testing result may be some higher than the actual long wire. However, the test data are still of importance and can be applied in the following research.

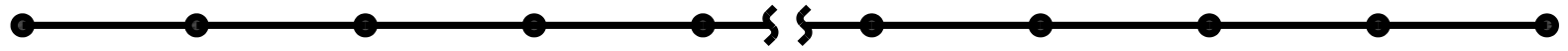

Fig. 1 Scheme sketches of individual wire series system

In Tab. 1, 81 tensile strength tests of $\varphi 7-1770 \mathrm{MPa}$ wires are given. All test specimen were $L_{0}=0.25 \mathrm{~m}$ long and $7 \mathrm{~mm}$ in diameter (widely used in China). According to statistical theory, the data sample size is large enough to perform the statistical analysis.

Tab. 1 Results of wire tensile strength tests

\begin{tabular}{ccc||ccc||ccc||ccc}
\hline Test & $\begin{array}{c}\text { Area } \\
\text { Testrength }\end{array}$ & \multicolumn{3}{|c||}{ Sest Area } & Strength & \multicolumn{3}{|c||}{ Area } & Strength & \multicolumn{3}{|c|}{ Area } & Strength \\
& $\left(\mathrm{mm}^{2}\right)$ & $(\mathrm{kN})$ & & $\left(\mathrm{mm}^{2}\right)$ & $(\mathrm{kN})$ & & $\left(\mathrm{mm}^{2}\right)$ & $(\mathrm{kN})$ & & $\left(\mathrm{mm}^{2}\right)$ & $(\mathrm{kN})$ \\
\hline 1 & 38.82 & 69.16 & 22 & 38.93 & 73.51 & 43 & 38.82 & 69.66 & 64 & 39.04 & 71.2 \\
2 & 39.15 & 70.73 & 23 & 38.82 & 70.81 & 44 & 38.82 & 69.27 & 65 & 39.04 & 69.16 \\
3 & 38.93 & 69.54 & 24 & 38.82 & 70.04 & 45 & 38.93 & 69.66 & 66 & 38.59 & 70.2 \\
4 & 38.7 & 68.50 & 25 & 38.7 & 69.66 & 46 & 38.93 & 70.81 & 67 & 38.82 & 70.66 \\
5 & 38.59 & 69.31 & 26 & 38.7 & 70.04 & 47 & 38.93 & 70.81 & 68 & 38.59 & 71.16 \\
6 & 38.59 & 69.62 & 27 & 38.82 & 69.27 & 48 & 38.82 & 71.20 & 69 & 38.93 & 71.58 \\
7 & 38.82 & 69.35 & 28 & 38.93 & 68.89 & 49 & 38.82 & 71.20 & 70 & 38.7 & 70.23 \\
8 & 38.93 & 69.54 & 29 & 38.93 & 70.81 & 50 & 38.82 & 71.58 & 71 & 38.7 & 68.77 \\
9 & 38.93 & 70.16 & 30 & 38.93 & 70.81 & 51 & 38.82 & 70.04 & 72 & 38.48 & 69.62 \\
10 & 38.82 & 69.35 & 31 & 38.82 & 69.27 & 52 & 38.82 & 71.97 & 73 & 38.93 & 69.46 \\
11 & 39.04 & 70.08 & 32 & 38.93 & 71.20 & 53 & 38.82 & 69.27 & 74 & 38.82 & 69.16 \\
12 & 38.59 & 69.35 & 33 & 38.93 & 71.58 & 54 & 38.82 & 69.66 & 75 & 38.82 & 72.35 \\
13 & 38.59 & 70.16 & 34 & 38.93 & 70.04 & 55 & 38.93 & 70.43 & 76 & 38.59 & 69.58 \\
14 & 38.7 & 69.16 & 35 & 38.93 & 70.04 & 56 & 38.82 & 70.43 & 77 & 38.7 & 70.85 \\
15 & 39.15 & 69.62 & 36 & 38.93 & 70.04 & 57 & 38.82 & 71.20 & 78 & 39.04 & 69.96 \\
16 & 38.82 & 70.12 & 37 & 39.04 & 69.27 & 58 & 38.82 & 70.43 & 79 & 39.15 & 70.97 \\
17 & 39.04 & 69.04 & 38 & 38.82 & 70.81 & 59 & 38.82 & 70.04 & 80 & 38.93 & 69.43 \\
18 & 38.93 & 69.35 & 39 & 38.93 & 70.43 & 60 & 38.7 & 70.04 & 81 & 38.7 & 68.85 \\
19 & 39.26 & 68.89 & 40 & 38.7 & 70.04 & 61 & 38.82 & 70.04 & & & \\
20 & 38.82 & 72.35 & 41 & 38.82 & 70.04 & 62 & 38.93 & 70.43 & & & \\
21 & 38.93 & 71.58 & 42 & 38.82 & 70.04 & 63 & 38.82 & 69.93 & & & \\
\hline
\end{tabular}


Due to the difficulty of theoretical study, a consensus on probabilistic distribution of single wire strength has not been reached. Nonetheless, according to the previous approaches and experience, there are still two different types widely used to model the behavior of it:

(1) Normal distribution assumption [4, 9]

$$
F_{Z}(z)=\Phi\left(\frac{z-\mu}{\sigma}\right)
$$

where $F_{Z}(z)$ is the normal distribution function, $\mu$ is the mean value and $\sigma$ is the standard deviation.

(2) Weibull distribution assumption [2,3]

$$
F_{Z}(z)=1-\exp \left[-\left(\frac{z}{\mu}\right)^{k}\right]
$$

with mean value

$$
E(Z)=\mu \Gamma(1+1 / k)
$$

and variation

$$
V(Z)=u^{2}\left[\Gamma(1+2 / k)-\Gamma^{2}(1+1 / k)\right]
$$

where $\Gamma(\cdot)$ is the Gamma function, $u$ is the scale factor and $k$ is the shape factor.

In order to verify the fitness of these two assumptions, a parameter estimation as well as goodness of fit tests will be performed hereinafter. Taking the data in Table 1 as basis, the distribution parameters $\mu, \sigma, u$ and $k$ in functions may be estimated by Moment Estimation and Maximum Likelihood Method respectively. After this procedure, two Kolmogorov- Smirnov tests

\begin{tabular}{|c|c|c|c|c|c|}
\hline \multirow{2}{*}{$\begin{array}{l}\text { Distribution } \\
\text { type }\end{array}$} & \multicolumn{3}{|c|}{ Parameters estimation } & \multirow{2}{*}{$\begin{array}{l}P \text { value of } K-S \\
\text { test }\end{array}$} & \multirow{2}{*}{$\begin{array}{l}K-S \text { tes } \\
\text { result }\end{array}$} \\
\hline & parameter & estimation & $\begin{array}{c}95 \% \text {-confidence } \\
\text { interval }\end{array}$ & & \\
\hline \multirow{3}{*}{$\begin{array}{l}\text { Normal } \\
\text { distribution }\end{array}$} & $\mu$ & 70.16 & {$[69.95,70.36]$} & \multirow{3}{*}{0.1942} & \multirow{3}{*}{ accept } \\
\hline & & & & & \\
\hline & $\sigma$ & 0.933 & {$[0.808,1.104]$} & & \\
\hline \multirow[t]{2}{*}{$\begin{array}{c}\text { Weibull } \\
\text { distribution }\end{array}$} & $u$ & 70.65 & {$[70.39,70.91]$} & \multirow[t]{2}{*}{0.0401} & \multirow[t]{2}{*}{ reject } \\
\hline & $k$ & 63.57 & {$[55.07,73.37]$} & & \\
\hline
\end{tabular}
(goodness of fit test) with significance level of 5\% are performed. The result may be seen in Table 2 and Fig. 2.

Tab. 2 Results of distribution parameters estimation and $K-S$ test for wires tensile strength 


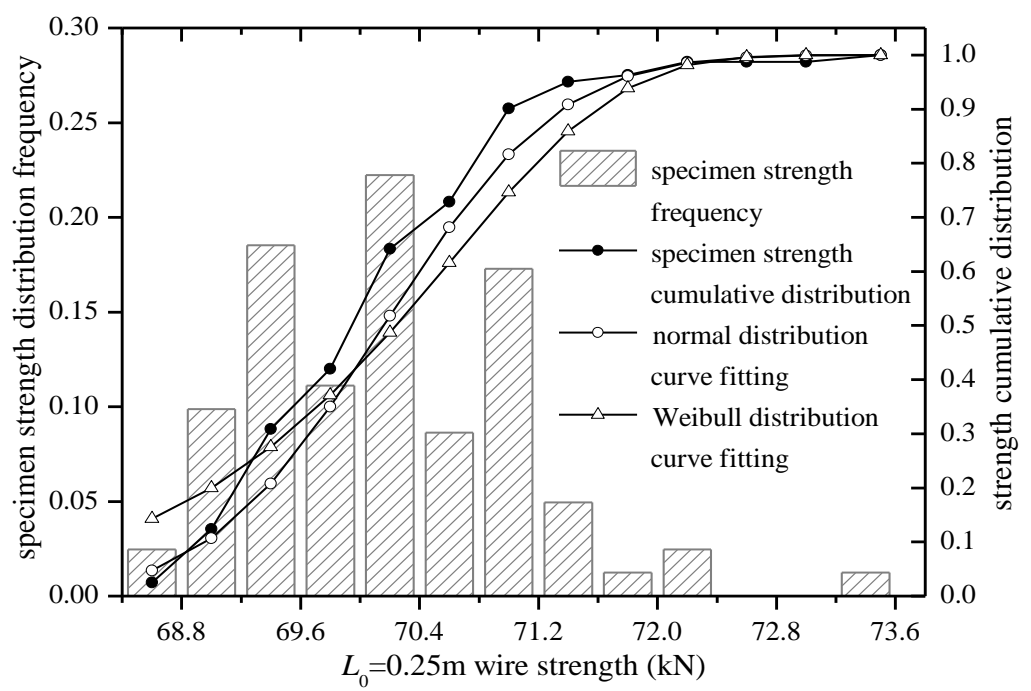

Fig. 2 Probability distribution parameters estimation of wires tensile resistance

According to the result of Kolmogorov-Smirnov tests, the normal distribution assumption passes the test, while the Weibull distribution assumption doesn't. Therefore, it seems more proper to select the former as the distribution type of short wire strength. The mean value and standard deviation of it are $70.16 \mathrm{kN}$ and $0.93 \mathrm{kN}$ respectively, which also indicates the coefficient of variation 0.013 .

The work above mainly focus on the strength of short wires i.e. length $L_{0}=0.25 \mathrm{~m}$. For the long wires in actual cables, the issue will be discussed hereinafter. As this type of wire is rather long, the material parameters and/or the defects within a certain length may be assumed to be correlated [2]. Define this length as the correlation length $L_{\rho}$, the expression may be

$$
L_{\rho}=\int_{0}^{\infty}\left|\rho_{Z Z}(\tau)\right| \mathrm{d} \tau
$$

where $\rho_{Z Z}(\tau)$ is the auto-correlation function for the wire strength. Then the number $\lambda$ of elements in the whole wire is given by

$$
\lambda=\frac{L}{L_{\rho}}=\frac{L}{l L_{0}}
$$

where $L$ is the length of long wires, $l$ is the ratio of correlation length $L_{\rho}$ to specimen length $L_{0}$. In general, $l$ is greater than 1 , which means the strength of wire in length $L_{\rho}$ also follow the normal distribution of $N(70.16,0.93)$.

Then for the long single wire, as its strength $Z *$ is determined by the weakest link, i.e

$$
\mathrm{Z}^{*}=\min (\mathrm{Z} 1, \mathrm{Z} 2, \ldots, \mathrm{Z \lambda})
$$

According to the series system theory, the cumulative distribution function of it may be

$$
\begin{aligned}
F_{Z}\left(z^{*}\right) & =1-P\left[\left(Z_{1}>z^{*}\right) \bigcap\left(Z_{2}>z^{*}\right) \bigcap \cdots \bigcap\left(Z_{\lambda}>z^{*}\right)\right] \\
& =1-\left(1-\Phi\left(\frac{z^{*}-70.16}{0.93}\right)\right)^{\lambda}
\end{aligned}
$$

Fig. 3 shows the relationship between the long wire strength and subsection number $\lambda$ in accordance with Eq.8. From the figure, a general law can be observed: with the wire length or subsection number $\lambda$ increasing, the strength of it may decrease. This phenomenon is generally called "the length effect". For long-span cable-stayed bridges, especially with super long cables, the effect should not be ignored. 


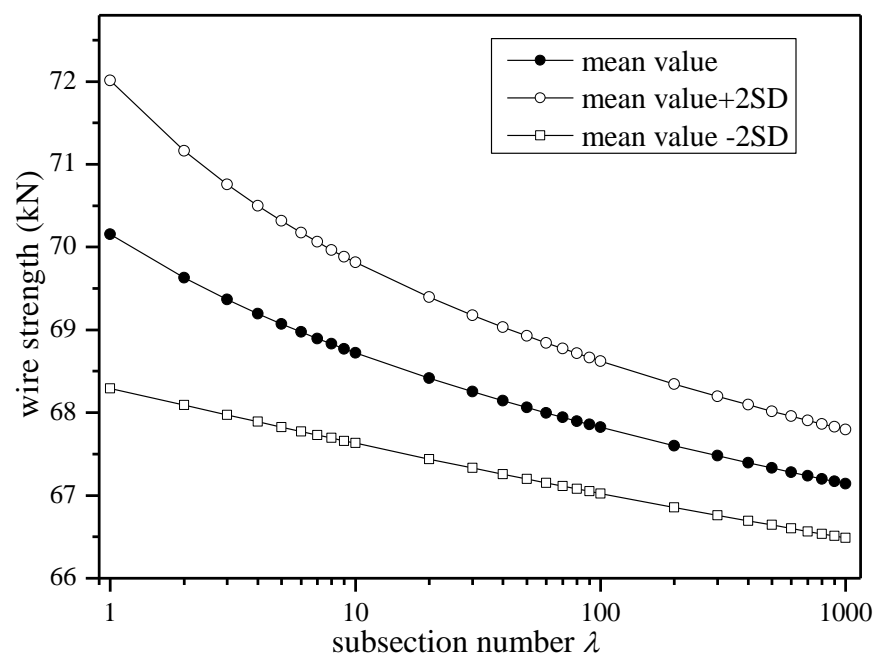

Fig. 3 Relationship between wire tensile resistance and subsection number $\lambda$

\section{Cable Mechanical Model and Load Bearing Estimation Method}

As the parallel wire cables are composed by mass parallel wires with protective outer layers, the mechanism of them can be regard as equal tensioning models. The bearing capacity of this parallel wire cables may be assessed by modeling the cable as a parallel system, see Fig. 4.

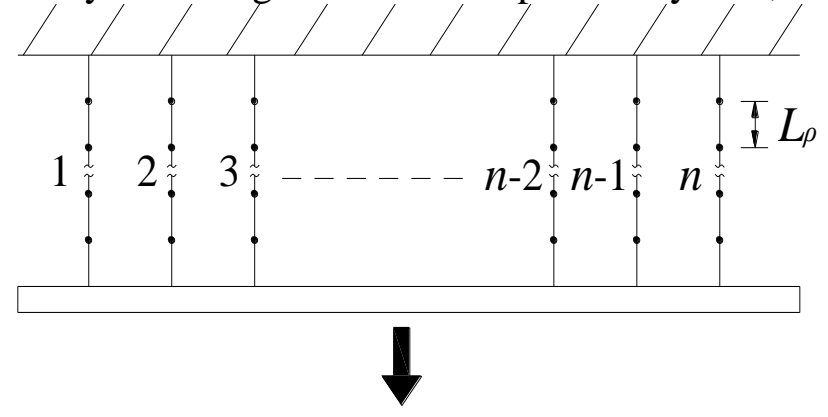

Fig. 4 Parallel system for the modeling of bundles of parallel wires

Failure of the wires may be ductile or brittle depending on the wire material characteristics. However, even for a ductile wire failure, the length of the plastic zone will be just in the order of 1-3 times the diameter of wire [2]. Hence it's easily noted that the elastic strains at failure will dominate and the wire failure always has a brittle characteristic [10]. According to these prerequisites, the Daniels probabilistic strength theory (hereinafter referred to as Daniels model) [11, 12] can be used to investigate the bearing capacity of the cables.

For the $n$ wire cable, firstly arrange each wire strength in a ascending order, and define them as $Z_{(1)} *, Z_{(2)} *, \ldots, Z_{(n)} *$. When $\mathrm{m}$ wires get fractured one after another, the left minimum wire strength will be $Z_{(m+1)}{ }^{*}$. At this stage, the bearing capacity of the cable can be appropriately expressed [10] as

\section{$F=\max \left((n-m) \times Z_{(m+1)} *\right)$}

As the strength of each wire is random variable, the final result of bearing capacity $F$ from Eq.9 may also be stochastic naturally. According to central limit theorem, if $n$ is large enough the bearing capacity $F$ may converge to be normal distributed. For cables in actual engineering, this conclusion is always valid due to the large numbers of cable wires.

Although the estimation equation has been given clearly, it is still extremely difficult to calculate it. Although there is some asymptotic solution, the precision may vary. On account of this situation, the Monte Carlo sampling (MCS) method is chosen to meet the requirements of precision and application. Using MCS a bundle of $139 \varphi 7-1770 \mathrm{MPa}$ wires is evaluated with the variation of subsection number $\lambda$.The result is shown in Fig. 5, each point is sampled for 100000 times. 


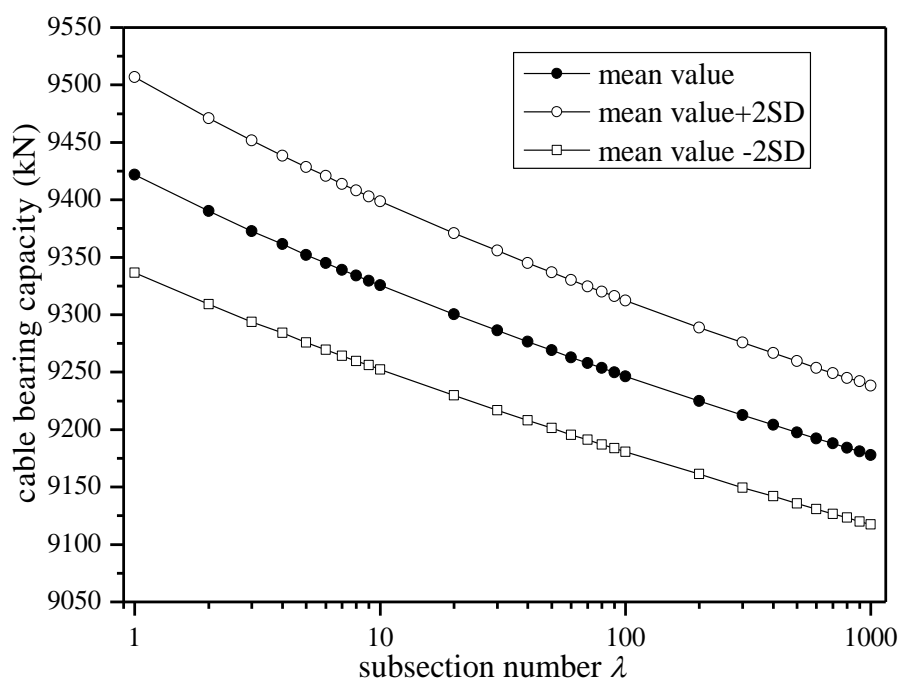

Fig. 5 Bearing capacity of cable as function of subsection number $\lambda$

In real engineering, the cables are generally offered with given length. The application of the present method may need correlation length $L_{\rho}$ to determine the subsection number $\lambda$. For this issue, according to the study by Castillo et al. [13] the value of $L_{\rho}$ may be taken as $1.96 \mathrm{~m}$, which is rather smaller in comparison to real cables. The situation may also indicate that a margin of error may exist inevitably without the consideration of length effect and the error makes the cable safety weaker.

According to the research above, the relationship between cable bearing capacity $F$ and wire number $n$ will be now discussed. As bearing capacity $F$ with more wires is always greater than that with less wires, the bearing capacity per wire will be defined as below for convenience.

$$
F_{\text {per wire }}=F / n
$$

Then the relationship between $F_{\text {per wire }}$ and wire number $n$ can be illustrated in Fig. 6 .

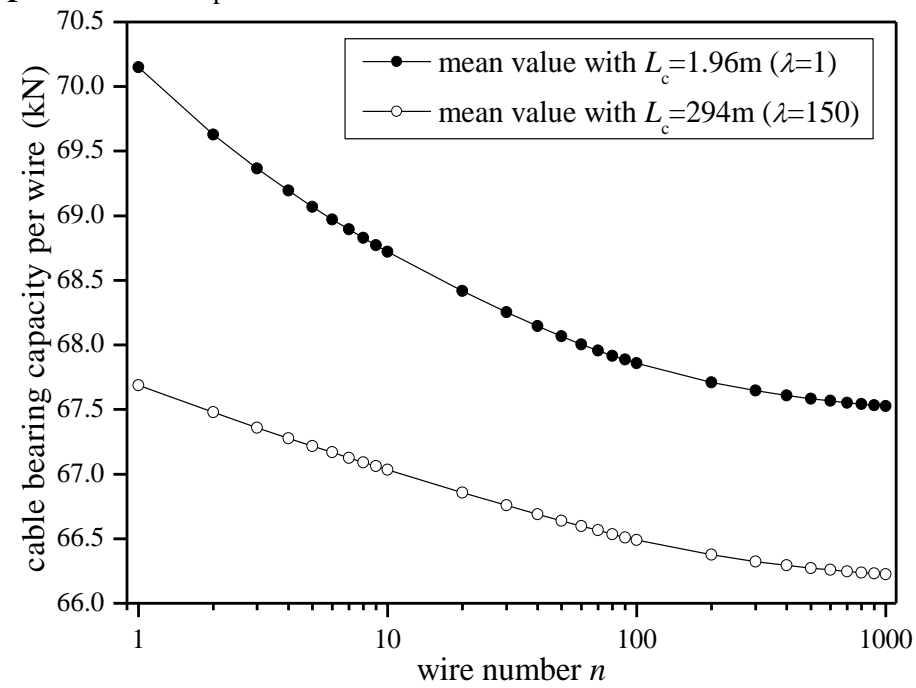

Fig. 6 Bearing capacity of single wire as function of the number of wires in the cable

In Fig. 6 it can be seen that the $F_{\text {per wire }}$ is decreasing with the increase of wire number $n$ in the considered case. And as the mean value of $F_{\text {per wire }}$ decreases, the standard deviation decreases even faster. Together the two effects are often referred to as the Daniels effect. However, a further study implies that the extent of Daniels effect varies for different cables in length. For short cables, the influence of Daniels effect seems more obvious, the $F_{\text {per wire }}$ reduction amount is greater than that of long cables. This phenomenon can be explained by the length effect: for individual wires, the coefficient of variation decreases with the increase of wire length and makes the strength of each 
wires uniform. Then the $F_{\text {per wire }}$ tends to be gentle at the macro level and Daniels effect gets weak. From this point of view, another conclusion may also be deduced that there exists a strong coupling characteristic between length effect and Daniels effect for the long stay-cables.

\section{Conclusions}

(1) For wire specimen $\left(L_{0}=0.25 \mathrm{~m}\right)$, according to test result and Kolmogorov-Smirnov test it seems more proper to select normal distribution as the distribution type of wire specimen strength.

(2) The present MCS based method can estimate cable bearing capacity effectively. Compared with previous asymptotic equations, this method has wide application and high precision.

(3) According to the length effect and Daniels effect, both the mean value and coefficient of variation of cable bearing capacity (per wire) will decrease with the increasing of wire number. For the long cables, the two effects even have a strong coupling characteristic.

\section{References}

[1] W.T. Wang. Cable Replacement Engineering of Cable-stayed bridge. China Communications Press, Beijing, 1997.

[2] M.H. Faber, S. Engelund and R. Rackwitz. Aspects of parallel wire cable reliability. Structural Safety, 2006, 25(2): 201-225.

[3] C.M. Lan, H. Li and Y. Ju. Bearing capacity assessment for parallel wire cables. China Civil Engineering Journal, 2013, 46(5): 31-38.

[4] P.E. Sante Camo. Probabilistic strength estimates and reliability of damaged parallel wire cables. ASCE Journal of Bridge Engineering, 2003, 8(5): 297-311.

[5] C. Cremona. Probabilistic approach for cable residual strength assessment. Engineering Structures, 2003, 25(3): 377-384.

[6] J.S. Zhu, and R.C. Xiao. A study on the safety assessment method for stay cables of long-span cable-stayed bridges. China Civil Engineering Journal, 2006, 39 (9): 74-79.

[7] W. Weibull. A statistical distribution function of wide applicability. Journal of Applied Mechanics, 1951, (9): 293-297.

[8] Board of Engineers. Standard of Hot-dip galvanized steel wires for bridge cables (GB/T 17101-2008). Standards Press of China, Beijing, 2008.

[9] J. Matteo, G. Deodatis and D.P. Billington. Safety analysis of suspension-bridge cables: Williamsburg bridge. ASCE Journal of Structural Engineering, 1994, 120(11): 3197-3211.

[10]J. Xu. Damage evolution mechanism and remained service lives evaluation of stayed cables. Ph.D. Thesis, Tongji University, shanghai, 2006.

[11]H.E. Daniels. The statistical theory of the strength of bundles of threads, Part I. Proceedings of the royal society, Series A. London, 1945: 405-435.

[12]S. Gollwitzer and R. Rackwitz. On the reliability of Daniels systems. Structural Safety, 1990, 7(2): 229-243.

[13]E. Castillo, M. López-Aenlle, A. Ramos, A. Fernández-Canteli, R. Kieselbach and V. Esslinger. Specimen length effect on parameter estimation in modeling fatigue strength by Weibull distribution. International Journal of Fatigue, 2006, 28(9): 1047-1058. 